\title{
Report of Rhombognathus scutulatus (Acari: Halacaridae) from Goa, India
}

\author{
TAPAS CHATTERJEE, ${ }^{1}$ MANDAR NANAJKAR ${ }^{2}$ \\ ${ }^{1}$ Crescent International School, Bario, Govindpur, Dhanbad 828109, Jharkhand, India \\ 2 CSIR, National Institute of Oceanography, Dona Paula, Goa-403004, India \\ Corresponding author, e-mails: drtchatterjee@yahoo.co.in,mandar@nio.org
}
Keywords Rhombognathus, halacarid mite, Goa
Abstract Rhombognathus scutulatus Bartsch is reported here from Goa, India. World distribution of this species is also provided.

Pierwsze stwierdzenie Rhombognathus scutulatus (Acari: Halacaridae) na Goa, Indie

Słowa kluczowe: Rhombognathus, wodopójki z grupy Halacaride, Goa

Streszczenie Artykuł prezentuje pierwsze stwierdzenie na Goa (zachodnie wybrzeże Indii) Rhombognathus scutulatus na tle rozmieszczenia gatunku na świecie.

\section{Introduction}

Halacarid mites of west coast of India were studied by the first author from Kerala (Chatterjee, Sarma, 1993; Chatterjee, 1995, 2000; Bartsch, Chatterjee, 2001), Maharastra (Chatterjee, Chang, 2004) and Goa (Sarma, Chatterjee, 1993; Chatterjee, 2015, 2018; Chatterjee, Guru, 2011a, b; Chatterjee, Marshall, Guru, Ingole, Pesic, 2012; Chatterjee, Guru, Sorensen, 2013). In the present communication we report Rhombognathus scutulatus Bartsch, 1983 from rocky shore of Goa. Rhombognathus scutulatus was first described from Philippines (Bartsch, 1983). In India, this species was recorded earlier from Andhra Pradesh, Kanya Kumari, Kerala and Andaman \& Nicobar Islands (Chatterjee, 1995).

\section{Material and Methods}

The material examined for the present study was collected from sediments of the intertidal seaweeds viz. Sargassum, Ulva and Gracillaria from Anjuna beach, Goa. After preliminary observation, one specimen was processed for SEM study. 


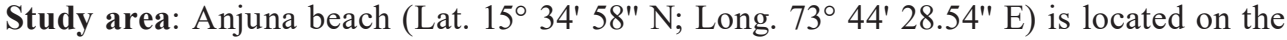
northern stretches of Goa state on the West Coast of India. The coast is mainly sandy and rocky shore. The rocky shore has small intertidal rock pools which are rich with diverse marine flora and fauna. The seaweed cover was seen on rocky shore and was more on seaward side represented by species of Sargassum, Hypnea, Spatoglossum, Chaetomorpha, Sphacealria, Cladophora, Dictyota, Gracillaria, Porphyra and Amphiroa. Some of the seaweeds like Sargassum sp. were found submerged in the water mainly in the splash zone area. The calcified genera include Amphiroa sp. \& Cheliosporum sp. were found in abundance in the rook pool area. Whereas the rocky areas display large flank of Sargassum in rock pools \& crevices. Three common seaweeds Sargassum, Ulva and Gracillaria were collected for the study of mites.

The following abbreviations are used in the text, table and figure legends: AE, anterior epimeral plate; PE, posterior epimeral plate; PGS, perigenital setae; SGS, subgenital setae.

\section{Result and Discussion}

\section{Rhombognathus scutulatus Bartsch 1983}

Rhombognathus scutulatus Bartsch (1983, pp. 413-415, figs. 46-57; 1993, pp. 20-21, figs. 1A-C; 1999, pp. 354-355, figs. 12F-H; 2000, p. 190; 2003, pp. 273-275, figs. 9A-D; 2006, pp. 42-43; 2009, pp. 35-36); Chatterjee (1995, pp. 284-285, figs. 15-19) Chatterjee and De Troch (2000, pp. 187-188, fig. 6); Smit (2011, p. 344); Abé and Etemadi (2014, pp. 15, 16, figs 23, 24).

Material Examined: Males and females from Goa - Anjuna beach among rocky algae Sargassum, Ulva and Gracillaria.

Brief Description: All dorsal plates fused in single shield and sculptured with foveae (Figure 1A). Posterodorsal area with a pair of setae. All ventral plates fused to a ventral shield (Figure 1B). AE area with 3 pairs of ventral setae plus one pair of adjunctive marginal setae. Each $\mathrm{PE}$ area with 3 ventral, one dorsal plus 1 adjunctive seta. Male with 11-12 pairs plumose PGS and 2 pairs SGS (Figure 1C). Female with 5 pairs PGS and 2 pairs SGS. Gnathostoma small and compact. Palp 4-segmented. Palpal patella $\left(\mathrm{P}_{2}\right)$ and trochanter without any setae. Palpal telofemur with 1 seta and tibiotarsus with 3 setae. Telofemora I and II with 6 setae ( 2 ventral and 4 dorsal); telofemora III and IV devoid of any ventral seta and bearing 3 dorsal setae. Tibia I with 5 setae of which 2 ventral pectinate. Legs with carpite on tarsi and devoid of median claw. Lateral claw endoplanate with broad accessory process bearing about 13-15 teeth (Figure 1D).

Distribution: This species is widely distributed in the south-western Pacific Ocean and Indian Ocean: Philippines, Singapore, Australia, Sri Lanka, India, Iran, Kenya, Mauritius and New Gunia. Table 1 and Figure 2 show the distribution of this species.

Remarks: There are six species of Rhombognathus viz. R. aspidotus Bartsch (2006), R. conjunctus Bartsch (1986), R. parvulus Viets (1939), R. peltatus Viets (1939), R. scutulatus Bartsch and $R$. similis Bartsch (1977) in which dorsal plates fused in single shield. $R$. similis was reported from Andaman \& Nicobar islands (Chatterjee, 1995) as $R$. similis may belong to other species and should be considered at present as Rhombognathus sp. Among Rhombognathus species in which dorsal plates fused in single shield, $R$ scutulatus and Rhombognathus sp. have been recorded from Indian Ocean. 


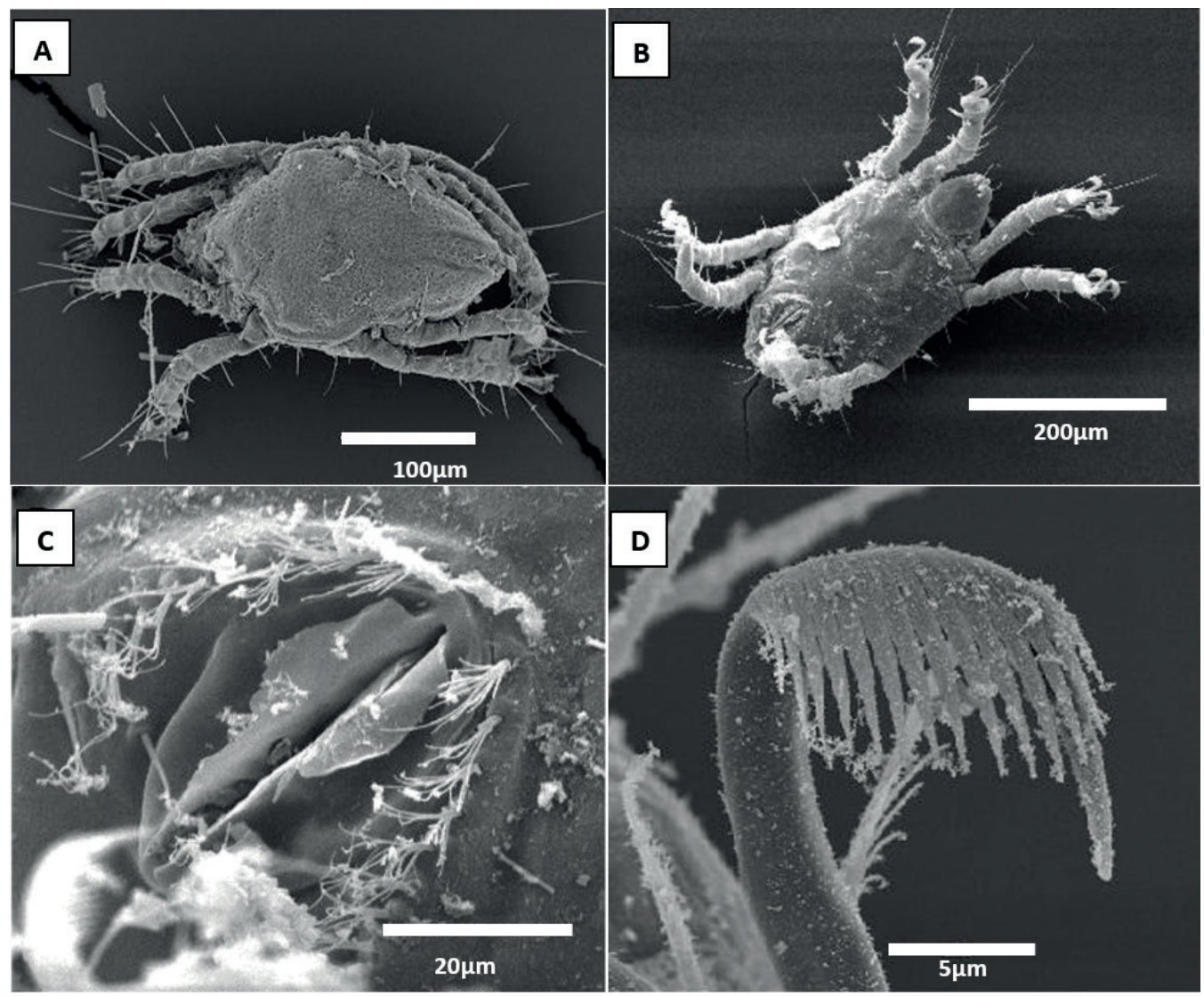

Figure 1. Rhombognathus scutulatus Bartsch, SEM figs. A. Dorsal view; B. Ventral view; C. Genital area of male showing PGS and SGS; D. Claw of tarsus III

Table 1. Rhombognathus scutulatus Bartsch: Localities with habitats

\begin{tabular}{|l|c|c|c|}
\hline \multicolumn{1}{|c|}{ Locality } & Habitat & Ocean & Reference \\
\hline 1 & 2 & 3 & 4 \\
\hline Philippines: Negros Island & $0-3 \mathrm{~m}$ & $\begin{array}{c}\text { PTW: Pacific Ocean, } \\
\text { tropical west }\end{array}$ & Bartsch (1983) \\
\hline $\begin{array}{l}\text { Australia: Rottnest Is - } \\
\text { Bickley point, Nancy cove, } \\
\text { Little Armstrong Bay, } \\
\text { western Australia }\end{array}$ & Amphibolis sp, Caulerpa sp. & $\begin{array}{c}\text { ISE: Indian Ocean, } \\
\text { south east }\end{array}$ & Bartsch (1993) \\
\hline $\begin{array}{l}\text { Australia: Rottnest Is - } \\
\text { Cape Vlamingh, Fish Hook } \\
\text { Bay western Australia }\end{array}$ & $\begin{array}{c}\text { Algae: Amphiroa sp. } \\
\text { Cystophora } \text { sp, Zonaria sp. }\end{array}$ & $\begin{array}{c}\text { ISE: Indian Ocean, } \\
\text { south east }\end{array}$ & Bartsch (1993) \\
\hline
\end{tabular}




\begin{tabular}{|c|c|c|c|}
\hline 1 & 2 & 3 & 4 \\
\hline $\begin{array}{l}\text { India: Palm beach, } \\
\text { Visakhapatnam, Andhra } \\
\text { Pradesh }\end{array}$ & Among rocky algae & $\begin{array}{l}\text { ITE: Indian Ocean, } \\
\text { tropical east }\end{array}$ & Chatterjee (1995) \\
\hline $\begin{array}{l}\text { India: Kanya Kumari } \\
\text { (=Cape comorin), Tamil } \\
\text { Nadu }\end{array}$ & Among rocky algae & $\begin{array}{l}\text { ITE: Indian Ocean, } \\
\text { tropical east }\end{array}$ & Chatterjee (1995) \\
\hline India: Kovalam, Kerala & Among rocky algae & $\begin{array}{l}\text { ITE: Indian Ocean, } \\
\text { tropical east }\end{array}$ & Chatterjee (1995) \\
\hline $\begin{array}{l}\text { India: Corvin cove, } \\
\text { Andaman \& Nicobar Islands }\end{array}$ & Among coralline algae & $\begin{array}{l}\text { ITE: Indian Ocean, } \\
\text { tropical east }\end{array}$ & Chatterjee (1995) \\
\hline $\begin{array}{l}\text { Australia: Rottnest Is - } \\
\text { Bickley Bay, Bickley point; } \\
\text { Nancy cove }\end{array}$ & $\begin{array}{c}\text { Sea grass Amphibolis and various } \\
\text { algae }\end{array}$ & $\begin{array}{l}\text { ISE: Indian Ocean, } \\
\text { south east }\end{array}$ & Bartsch (1999) \\
\hline $\begin{array}{l}\text { Australia: Great Barrier } \\
\text { Reef, Cape Fergusen, AIMS } \\
\text { beach }\end{array}$ & Algae at low tide mark & $\begin{array}{l}\text { PTW: Pacific Ocean, } \\
\text { tropical west }\end{array}$ & Bartsch (2000) \\
\hline $\begin{array}{l}\text { Australia: Great Barrier } \\
\text { Reef, Magnetic Island, } \\
\text { Alma Bay }\end{array}$ & Rocky littoral algae & $\begin{array}{l}\text { PTW: Pacific Ocean, } \\
\text { tropical west }\end{array}$ & Bartsch (2000) \\
\hline Kenya: Gazi Bay & $\begin{array}{l}\text { Among sea grass: Thalassia } \\
\text { hemprichii, Halophila ovalis, } \\
\text { H. stipulacea, Halodule wrightii, } \\
\text { Syringodium isoetifolium }\end{array}$ & $\begin{array}{l}\text { ITW: Indian Ocean, } \\
\text { tropical west }\end{array}$ & $\begin{array}{l}\text { Chatterjee } \\
\& \text { De Troch } \\
\text { (2000) }\end{array}$ \\
\hline $\begin{array}{l}\text { Australia: Dampier, } \\
\text { northwestern Australia }\end{array}$ & $\begin{array}{c}\text { Padina sp. (Phaeophyta), } \\
\text { low water edge }\end{array}$ & $\begin{array}{l}\text { ITE: Indian Ocean, } \\
\text { tropical east }\end{array}$ & Bartsch (2003) \\
\hline $\begin{array}{l}\text { Australia: } 40 \text { Mile Beach } \\
\text { north of Cape Preston }\end{array}$ & brown algae Padina and Sargassum & $\begin{array}{l}\text { ITE: Indian Ocean, } \\
\text { tropical east }\end{array}$ & Bartsch (2003) \\
\hline $\begin{array}{l}\text { Australia: East coast } \\
\text { of the Burrup } \\
\text { Peninsula, Watering Cove }\end{array}$ & turf of small red algae & $\begin{array}{l}\text { ITE: Indian Ocean, } \\
\text { tropical east }\end{array}$ & Bartsch (2003) \\
\hline $\begin{array}{l}\text { Singapore: Strait } \\
\text { of Singapore, Labrador park }\end{array}$ & Small brown and green rocky algae & $\begin{array}{l}\text { PTW: Pacific Ocean, } \\
\text { tropical west }\end{array}$ & Bartsch (2006) \\
\hline Srilanka: Ahangama & $\begin{array}{l}\text { Among Halimeda from a moderately } \\
\text { exposed fringing reef flat }\end{array}$ & $\begin{array}{l}\text { ITE: Indian Ocean, } \\
\text { tropical east }\end{array}$ & Bartsch (2006) \\
\hline $\begin{array}{l}\text { Mauritius: South of Port } \\
\text { Louis, Flic en Flac }\end{array}$ & Intertidal wave exposed rocky shore & $\begin{array}{l}\text { ITW: Indian Ocean, } \\
\text { tropical west }\end{array}$ & Bartsch (2009) \\
\hline $\begin{array}{l}\text { New Gunia: Base G beach, } \\
\text { Jayapura }\end{array}$ & Marine littoral & $\begin{array}{l}\text { PTW: Pacific Ocean, } \\
\text { tropical west }\end{array}$ & Smit (2011) \\
\hline $\begin{array}{l}\text { Iran: Chabahar Beach, } \\
\text { Darya Bozorg, } \\
\text { Gulf of Oman }\end{array}$ & Sargassum $\mathrm{sp}$ at $1 \mathrm{~m}$ depth & $\begin{array}{l}\text { ITW: Indian Ocean, } \\
\text { tropical west }\end{array}$ & $\begin{array}{l}\text { Abe \& Etemadi } \\
\text { (2014) }\end{array}$ \\
\hline India: Goa & Various rocky algae & $\begin{array}{l}\text { ITE: Indian Ocean, } \\
\text { tropical east }\end{array}$ & Present report \\
\hline
\end{tabular}




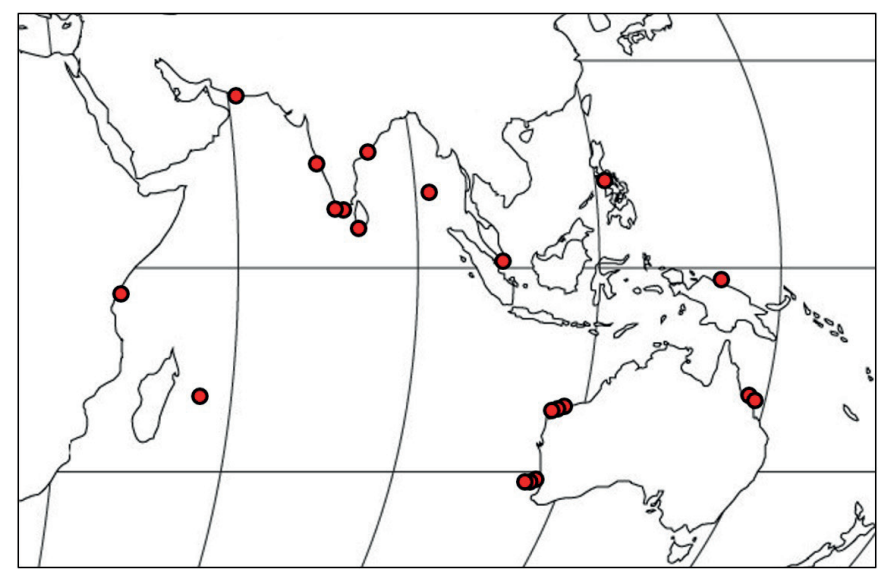

Figure 2. Distribution map of Rhombognathus scutulatus Bartsch

Chatterjee (1996) reported ciliate infestation on $R$. Scutulatus from Kovalam beach, Kerala.

Bartsch (2009) has given a comparative view on the variation in number of tines on the accessory process of lateral claws and length of idiosoma. This species exhibits wide variation in number of tines on the accessory process of lateral claws ranging from 10 to 28 (Bartsch, 2009; Abé, Etemadi, 2014).

\section{Acknowledgement}

Thanks are due to Dr. Martin V. Sorensen, Natural History Museum of Denmark, University of Copenhagen, Denmark for making the SEM photographs.

\section{References}

Abé, H., Etemadi, I. (2014). Two rhombognathine mites (Acari: Halacaridae) from the Gulf of Oman, Iran. Persian Journal of Acarology, 3 (2), 107-119.

Bartsch, I. (2006). A new species and new record of Rhombognathus from Singapore (Acari: Halacaridae). Zootaxa, 1120, 41-49.

Bartsch, I. (1999). Halacaridae (Acari) from Rottnest Island, Western Australia. Mites on fronds of the seagrass Amphibolis. In: D.I. Walker, F.E. Wells (eds.), The Seagrass Flora and Fauna of Rottnest Island, Western Australia (pp. 333-357). Perth: Western Australian Museum.

Bartsch, I. (2000). Rhombognathinae (Acari: Halacaridae) from the Great Barrier Reef, Australia. Memoirs of the Queensland Museum, 45, 165-203.

Bartsch, I. (2003). Rhombognathine mites (Halacaridae: Acari) from Dampier, Western Australia: taxonomy and biogeography. In: F.E. Wells, D.I. Walker, D.S. Jones (eds.), The Marine Flora and Fauna of Dampier, Western Australia (pp. 255-280). Perth: Western Australian Museum.

Bartsch, I. (1993). Rhombognathine mites (Halacaridae, Acari) from Rottnest Island, Western Australia. In: F.E. Wells, D.I. Walker, D.S. Jones, H. Kirkman, R. Lethbridge (eds.), The Marine Flora and Fauna of Rottnest Island, Western Australia (pp. 19-43). Perth: Western Australian Museum. 
Bartsch, I. (2009). Rhombognathus (Halacaridae: Rhombognathinae) from Mauritius, new records from the western Indian Ocean. Mitteilungen aus dem Hamburgischen Zoologischen Museum und Institut, 106, 27-38.

Bartsch, I. (1983). Zur Halacaridenfauna der Philippinen Beschreibung von fünf Arten der Gattung Rhombognathus (Acari, Halacaridae). Entomologische Mitteilungen aus dem Zoologischen Museum Hamburg, 7, 399-416.

Bartsch, I., Chatterjee, T. (2001). A new species of the Agauopsis brevipalpus group from India (Acari, Halacaridae). Entomologische Mitteilungen aus dem Zoologischen Museum Hamburg, 13 (164), 32-327.

Chatterjee, T. (1996). Ciliate infestation on phytal Halacaridae (Acari) from the Kovalam beach (Kerala coast). Journal of the Bombay Natural History Society, 93, 107-108.

Chatterjee, T. (2018). New record of Copidognathus andhraensis (Acari, Halacaridae) from the west coast of India, Arabian Sea. Acta Biologica, 25, 123-129.

Chatterjee, T. (1995). Record of three species of Rhombognathus (Halacaridae: Acari) from Indian Ocean region. Journal of the Bombay Natural History Society, 92, 282-286.

Chatterjee, T. (2015). Scanning electron microscopic observations of Copidognathus balakrishnani Chatterjee (Acari: Halacaridae) with notes on species of the Copidognathus balakrishnani group. Acta Biologica, 22, 213-222.

Chatterjee, T. (2000). Two new species of Copidognathus (Halacaridae: Acari) from Kerala. Journal of the Bombay Natural History Society, 97 (2), 235-239.

Chatterjee, T., Chang, C.Y. (2004). Two new Copidognathus halacarids (Acari: Halacaridae) from Mubai coast of India (the Arabian Sea). Italian Journal of Zoology, 71, 265-269.

Chatterjee, T., De Troch, M. (2000). Halacaridae (Acari) from Gazi Bay (Kenya): description and biogeography of three new and two known species. Hydrobiologia, 427, 177-194.

Chatterjee, T., Guru, B.C. (2011a). Scanning electron microscopic observations of Agauopsis arabia Bartsch \& Chatterjee (Acari, Halacaridae) with notes on the distribution of Agauopsis in the Indian Ocean. Natura Montenegrina, 10 (4), 415-423.

Chatterjee, T., Guru, B.C. (2011b). Scanning electron microscopic observation of Copidognathus arabicus Chatterjee \& Chang (Acari, Halacaridae) and notes on the distribution of the Copidognathus bairdi group sensu lato in the Indian Ocean. Natura Montenegrina, 10 (4), 425-434.

Chatterjee,T., Sarma, A.L.N. (1993). Occurrence of Copidognathus sideus Bartsch 1982 (Halacaridae: Acari) from Indian coast. Journal of the Bombay Natural History Society, 90 (2), 304-308.

Chatterjee, T., Marshall, D.J., Guru, B.C., Ingole, B, Pesic, V. (2012). A new species of the genus Acarothrix (Acari, Halacaridae) from Brunei Darussalam and India. Cahiers De Biologie Marine, 53 (4), 541-546.

Chatterjee, T., Guru, B.C., Sorensen, M.V. (2013). Report of Acarothrix palustris Bartsch (Acari: Halacaridae) from the Indian Ocean, Acta Biologica, 20, 17-26.

Sarma, A.L.N., Chatterjee, T. (1993). Occurrence of Arhodeoporus bonairensis (Viets, 1936) from Indian Ocean with zoogeographical remarks on genus Arhodeoporus Newell. Journal of the Bombay Natural History Society, 90 (3), 417-422.

Smit, H. (2011). New species of water mites from New Guinea (Acari: Hydrachnidia, Halacaridae). Acarologia, 51 (3), 321-345.

Cite as: Chatterjee, T., Nanajkar, M. (2019). Report of Rhombognathus scutulatus (Acari: Halacaridae) from Goa, India. Acta Biologica, 26, 127-132. DOI: 10.18276/ab.2019.26-11. 